\title{
Power optimisation using intelligent Clock gating dedicated for block RAM cascading technique in FPGA design.
}

gurwinder singh ( $\sim$ gurwindersingh87@gmail.com )

Guru Nanak Dev Engineering College

Munish Rattan

Guru Nanak Dev Engineering College

Gurjot Kaur Walia

Guru Nanak Dev Engineering College

\section{Research Article}

Keywords: Vivado Design suite, Field program gate array (FPGA), power optimization, Clock gating

Posted Date: September 10th, 2021

DOI: https://doi.org/10.21203/rs.3.rs-878601/v1

License: (c) (i) This work is licensed under a Creative Commons Attribution 4.0 International License.

Read Full License 
Noname manuscript No.

(will be inserted by the editor)

\title{
Power optimisation using intelligent Clock gating dedicated for block RAM cascading technique in FPGA design.
}

\author{
Gurwinder Singh $\cdot$ Munish Rattan · Gurjot Kaur Walia
}

Received: date / Accepted: date

\begin{abstract}
The current trend is the combination of chip size reduction and an increase in the number of circuits on chips has provided significant growth in battery consumption and critical energy efficiency leading to growth in the emerging Low Power Electronics sector. Our paper is committed to optimizing the power by eliminating cascading in block RAM. It dominates the amount of power dissipated in SOCs (System on Chips). High-level integration (HLS) allows hardware designers to think logically and not worry about low-level, cyclical details. It arranges the capability to quickly access the slot of design and the tradeoff between resource utilization and operation. Field Programmable Gate Arrays (FP- GAs) show significant progress in measuring speed and capacity to create a platform for the use of digital circuits. In the design of the FPGA, integration tools are used that perform various mitigation and improvement strategies. Integration tools utilize the RTL representation of a project with time constraints and generate a network list of the same level. Today, the advanced Xilinx Vivado Design Suite is used for FPGA design as a blending tool. In some cases, the Xilinx Vivado is unable to meet the required designer delays and power constraints. Therefore the primary goal of this paper is to optimize the power in design constraints in the Xilinx Vivado software.
\end{abstract}

Keywords Vivado Design suite, Field program gate array (FPGA), power optimization, Clock gating.

Mathematics Subject Classification (2020) MSC 94D9405,

1. Introduction: - Nowadays, in modern digital chips, reduced feature size and a higher clock frequency on the devices are required, power consumption is at its peak in today's integrated digital circuits [1]. Clock gating consumes up to 50-70\% of the total chip power and is expected to increase significantly in

\section{Gurwinder Singh}

M.Tech Student, Department of ECE, Guru Nanak Dev Engineering College, Punjab, Ludhiana, ndia

E-mail: gurwindersingh87@gmail.com

Munish Rattan

Department of ECE, Guru Nanak Dev Engineering College, Punjab, Ludhiana, India

Gurjot Kaur Walia

Department of ECE, Guru Nanak Dev Engineering College, Punjab, Ludhiana, India 
the following production designs. Therefore, reducing the power of the clock is very important. Clock gating is the key to the power reduction used by many Designers who also often use gate-level power integration tools [2].

In digital circuits, power is a combination of dynamic power and static power. Here, Dynamic power consumed by a clock signal, input/output signal, logic signal switching activities. Static power in the digital circuits consumes unnecessary static objects as an idle part of a device, where power dissipates due to leakage. Power consumption of the device is determined by the frequency of the operating clock (f) [3], node capacitance (C)[4], device operating voltage (V), and function $(\alpha)$ on various nodes in design. Most of the designs, above parameters, are set according to the device technology (e.g., voltage) or by design requirements (e.g., operating frequency).

However, several knobs in the design do not affect the device's output; still, they reach toggle. It forms an integral part of energy consumption. Vivado analyses the project, including third-party IP assets, of energy-saving potential to create a fine-grained clock gating with logic gating signals to reduce the unnecessary switching. This paper aims to optimise the power by using clock gating dedicated to block RAM cascading power optimisations.

1.1. Intelligent Clock gating:-Vivado design tools offer a wide range of power consumption to reduce dynamic energy use up to $30 \%$ on your design [5]. This adjustment is based on the ASIC clock style strategies to minimise energy consumption on project components that do not contribute to chip functioning and do not require a clock cycle.

In the Vivado design suite, Power optimisation implemented on the entire design and specified part of the design, which is done by an intelligent clock gating system. as shown in figure 1.1 power optimisation for the whole design[6] and figure 1.2 power optimisation for selected design components[6] of the plan.

1.2. Smart Clock gating BRAM enables functionality:- The function of intelligent clock gating dissipates the power for committed block RAM in simple dual-port mode or true dual-port mode as shown in the 1.3 figure and 1.4. These blocks grant many enabled; an array enables, write is enabled, and the output register clock is enabled, where Most of the energy-saving comes from the "an array enable." Software uses functionality to reduce power when there is no data to record and when there is no source of output
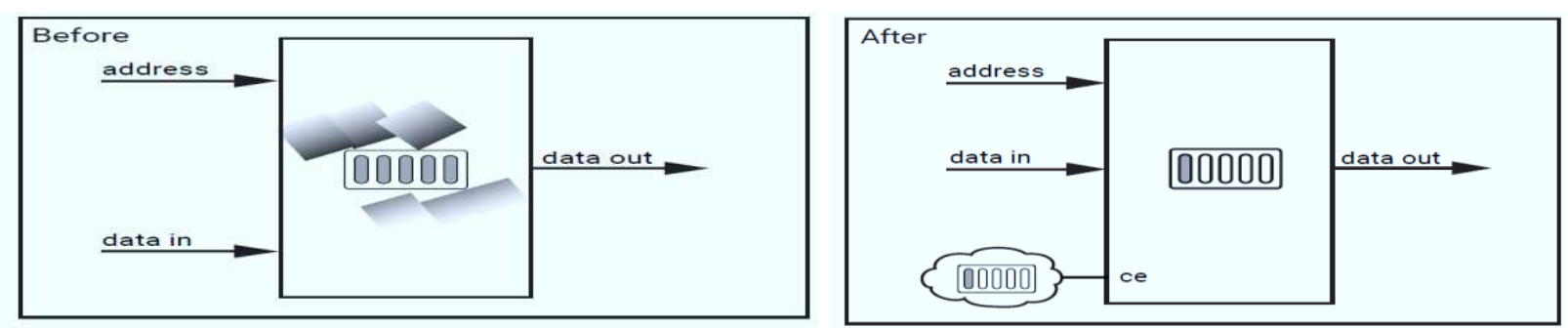

Figure 1.3 Clock gating BRAM enables [7]

An intelligent clock does not change the user's logic but instead builds an additional sense of gating logic. Therefore, the performance of the design is maintained at all times. However, this optimisation 
can also affect the timing, primarily if the application is used in critical means.

1.2.1. Block RAM Cascading Optimization. It is based on terminology that only one block RAM is activated during the operation[8].so, some block RAM can be disabled based on activity[9]. This saves up to 50-60 per cent of power on BRAM cascading. This adjustment is being made automatically in the opt_design section in the Vivado® Design Suite.

2. System design:- Here, in the system design target device is Xilinx Zynq platform. The Xilinx tool contains the Vivado design suite having part of the hardware to convert into a faster system. Hardware design using the Vivado design suite by configuring Xilinx IP cores and sample generator for test signal. Here the custom IP sends data to AXI video output IP[10] core. Figure 2.1 illustrated the basic flow of hardware design. Here. The sample data transfer signal to AXI steam to video out processed to zedboard board VGA here, the system act as a video processing platform.

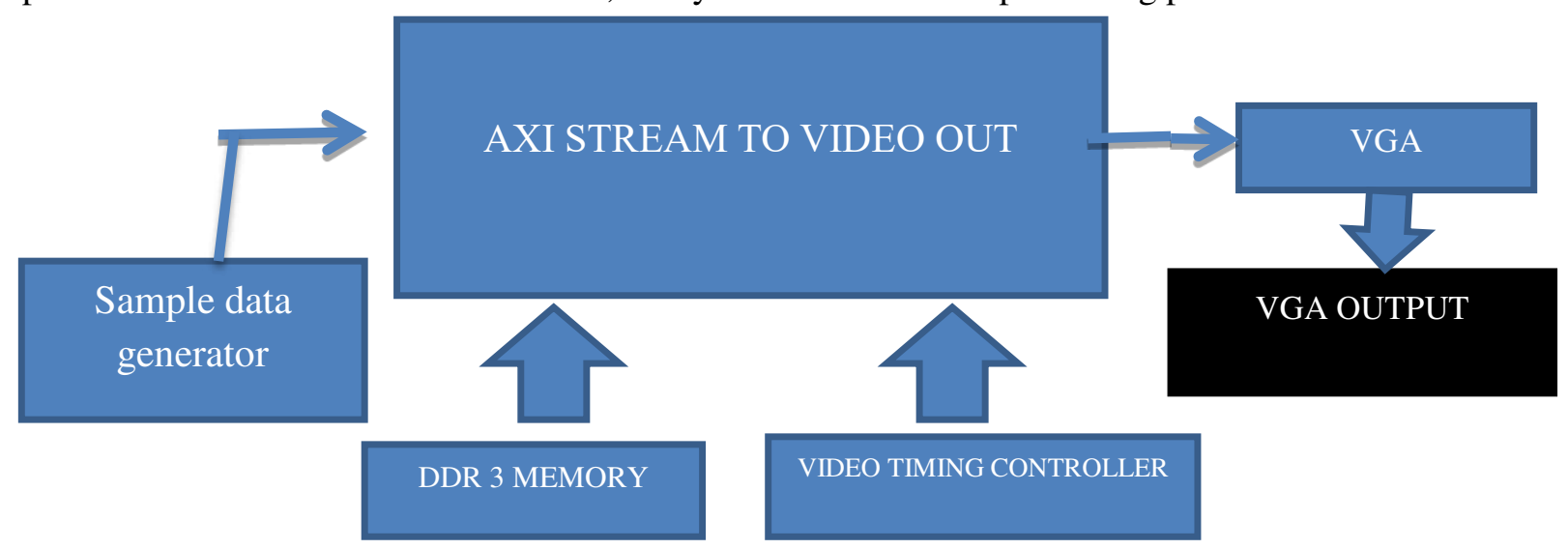

Figure 2.1 basic hardware design

Vivado's design suite analyses the design and generates reports based on activities such as power, utilisation, timing, DRC.

3. Result discussion:- Hardware implementation on Vivado design suite perform video processing is shown 4.1 where the sample data generator send data to AXI video stream out, and process to VGA output and power report and power optimisation report analysed below.

3.1. Power analysis and optimisation:- Vivado design suite allows the user to analyse the power report that helps identify and evaluate the hierarchy/resource with high capacity used in the project.

Figure 3.1.1 shows the power analysis report of design here maximum power consumed by the

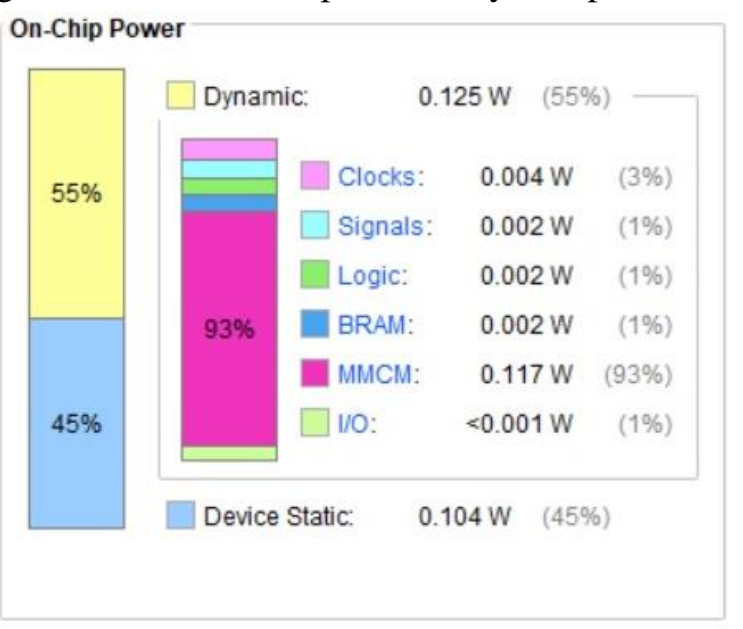

Figure 3.1.1 Non Optimized Power Analysis Report

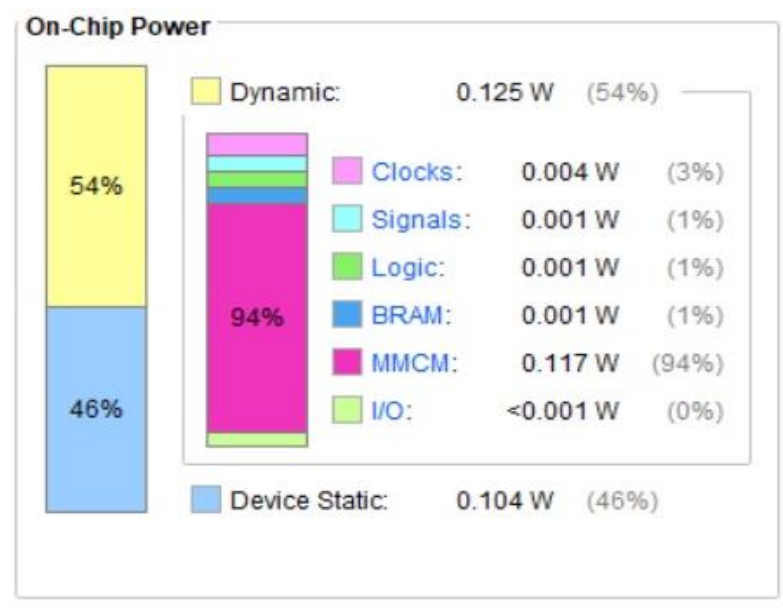

Fig:-3.1.2 Optimized Power Analysis Report) 
system is $0.23 \mathrm{w}$. After implementing the design on Zed board, we have analysed the total chip power as $0.23 \mathrm{~W}$. Here power used by BRAM is $0.002 \mathrm{~W}$. The dynamic and static power also analysed from simulation files are $0.125 \mathrm{~W}$ and $0.104 \mathrm{~W}$ of total chip power; after that, the next step is to reduce the power consumption generated by following steps implementation settings $\rightarrow \mathrm{Opt}$ Design $\rightarrow$ enable Power Opt Design $\rightarrow$ ok $\rightarrow$ run power report optimisation[11].

In the Summary view of the optimised power Report as shown in figure 3.1.2, observe BRAM power consumption reduced to $0.001 \mathrm{~W}$ from $0.002 \mathrm{~W}$ compared to the non-optimised power. Vivado design suite performs this action by intelligent clock gating BRAM cascading methods. So unnecessary components that consume power are cascaded, as shown in figure 3.1.3.

\begin{tabular}{|c|c|c|c|c|}
\hline Elements & Total & $\begin{array}{c}\text { User } \\
\text { Gated }\end{array}$ & $\begin{array}{c}\text { Tool } \\
\text { Gated }\end{array}$ & $\begin{array}{c}\text { Gated } \\
\text { (Total) }\end{array}$ \\
\hline $\begin{array}{c}\text { Number of } \\
\text { BRAMs }\end{array}$ & 1 & 1 & 0 & $\begin{array}{c}100.00 \\
0\end{array}$ \\
\hline $\begin{array}{c}\text { Number of } \\
\text { SRLs }\end{array}$ & 1 & 0 & 0 & 0.000 \\
\hline $\begin{array}{c}\text { Number of } \\
\text { Slice Resisters }\end{array}$ & 356 & 277 & 0 & 77.809 \\
\hline $\begin{array}{c}\text { Number of } \\
\text { XPM URAMs }\end{array}$ & 0 & 0 & 0 & 0.000 \\
\hline $\begin{array}{c}\text { BRAM write } \\
\text { mode changes }\end{array}$ & 3 & 0 & 0 & 0.000 \\
\hline
\end{tabular}

Figure 3.1.3 power optimisation report

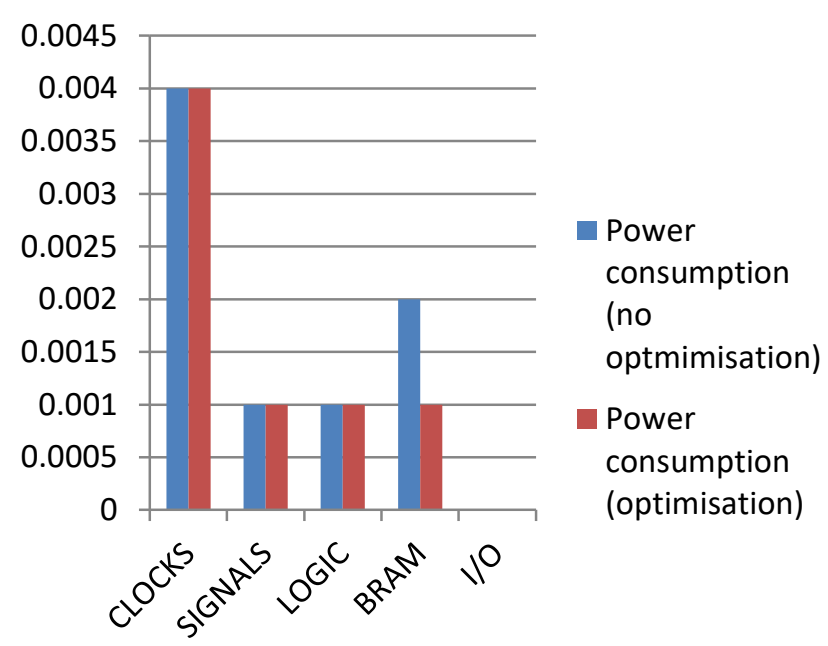

Vivado design suite also offers different environment settings. This experiment was done at a junction temperature of $27.6^{\circ} \mathrm{C}$ and a thermal margin of $57.4^{\circ} \mathrm{C}(4.8 \mathrm{~W})$ settings.

4. Conclusion and future work: - This analyses the performance of power consumption or optimise it to a certain level in video sources. This research work is helpful in designing technological devices that aim to reduce energy consumption and meet time requirements. The effectiveness of components is tested in natural environments. However, power consumption is reduced by cutting off unnecessary components or logic from the system. In summary, It should also see how energy analysis can be used to calculate the minimum energy requirement or find differences that meet the research needs. In addition, the work presented in this paper also expands by analysing this at different environmental conditions and reducing distortion or using different IPs in other ways [12]. In addition, this analysis is a significant guide to the power analysis of video processing systems as part of the future work of this forum. Moreover, another processing algorithm can be implemented in a 
structured concept using VIVADO.

Acknowledgement:- The authors would like to thank Dr. Narwant Singh Grewal (Head of department) Guru Nanak Dev Engineering College, Ludhiana.

\section{References}

[1] Gunjan Shrivastava, Shivendra Singh (2014) "Power Optimisation of Sequential Circuit Based ALU Using Gated Clock \&amp; Pulse Enable Logic” IEEE International Conference on Computational Intelligence and Communication Networks.

[2] Jitesh Shinde, Dr.S.S. Salankar(2011) “Clock gating -A Power Optimizing Technique for VLSI Circuits” IEEE, 2011 Annual IEEE India Conference.

[3] Tarek Darwish, Magdy Bayoumi (2005) “Trends in Low-Power VLSI Design",The Electrical Engineering Handbook.

[4] Ivan Ratkovic, Veljko Milutinovic (2015) "An Overview of Architecture-Level Power- and EnergyEfficient Design Techniques" Advances in Computers, 2015.

[5] Jairam S, Madhusudan Rao, Jithendra Srinivas, Parimala Vishwanath, Udayakumar H, Jagadish Rao (2008) "Clock Gating for Power Optimization in ASIC Design Cycle Theory \& Practice" Texas Instruments, India.

[6] Vivado Design Suite User Guide (2020) “Power Analysis and Optimization” UG907 (v2020.1) June 3, 2020,pp-72.

[7] User Guide (2016) “Vivado Design Suite power Analysis and Optimisation" UG907 (v2016.1) June 3, 2020,pp-72.

[8] Srinivasa Kolluri (2015) “UltraScale Architecture Low Power Technology Overview" WP451 (v1.1), October 15, 2015, pp-6-8.

[9] Vivado design suite tutorial (2018) "power analysis and optimisation" UG 997(v2018.2) June 6, 2018, pp85.

[10] User manual (2017) “AXI4-Stream to Video Out LogiCORE IP Product Guide” Vivado Design Suite, PG044 October 4, 2017, v4.0.

[11] Vivado Design Suite (2017) “AXI Reference Guide” UG1037 (v4.0) July 15, 2017.

[12] Naresh Grover, Dr. M. K. Soni, "Reduction of power consumption in FPGAs - an overview," I. J. Information Engineering and Electronic

Business, vol. 5, pp. 50-69, 2012. 
List of Figures:-

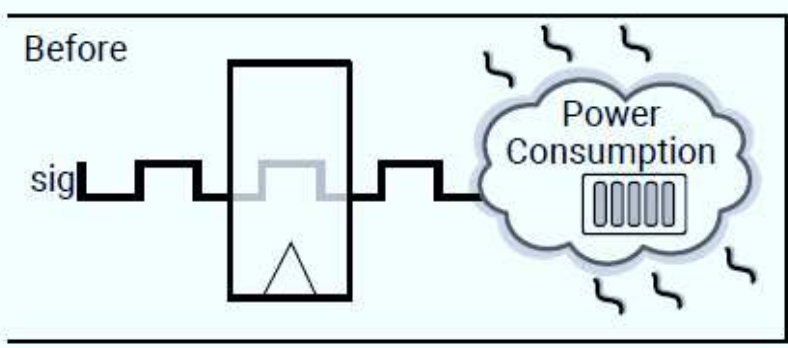

Figure 1.1 intelligent clock gating on whole system

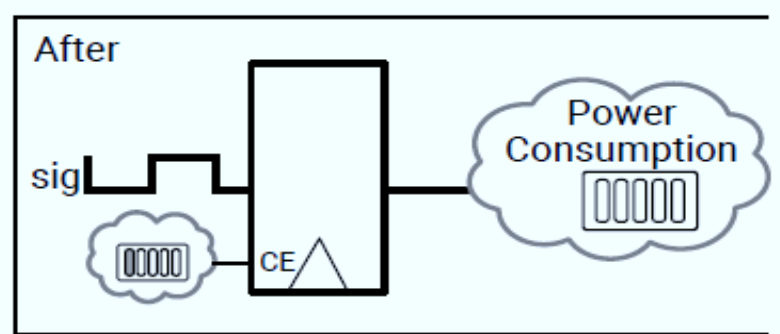

Figure 1.2 intelligent clock gating selected design components part.



Figure 3.1 video processing AXI stream video out view 
